\title{
Décadrages Décadrages
}

cinéma, à travers champs Cinéma, à travers champs

21-22| 2012

Cinéma élargi

\section{Paracinéma, Flicker et 3D : entretien avec Ken Jacobs}

\section{François Bovier et Adeena Mey}

Traducteur : Sylvain Portmann

\section{OpenEdition \\ Journals}

Édition électronique

URL : https://journals.openedition.org/decadrages/682

DOI : $10.4000 /$ decadrages. 682

ISSN : 2297-5977

Éditeur

Association Décadrages

Édition imprimée

Date de publication : 15 décembre 2012

Pagination : 124-130

ISBN : 978-2-9700668-5-9

ISSN : 2235-7823

\section{Référence électronique}

François Bovier et Adeena Mey, «Paracinéma, Flicker et 3D : entretien avec Ken Jacobs », Décadrages [En ligne], 21-22 | 2012, mis en ligne le 30 octobre 2014, consulté le 03 avril 2022. URL : http:// journals.openedition.org/decadrages/682 ; DOI : https://doi.org/10.4000/decadrages.682 


\section{Paracinéma, Flicker et 3D: entretien}

\section{avec Ken Jacobs}

\section{par François Bovier et Adeena Mey}

\section{Décadrages}

Il y a une étroite interaction entre votre œuvre filmique et une réflexion sur la construction des modes de représentation historiques (et idéologiques). En un sens, nous pourrions parler d'une tension fructueuse entre des idées conceptuelles et des actes performatifs qui mobilisent le corps.

Vous avez forgé la notion de "paracinéma" pour décrire vos performances avec le Nervous System. Pourriez-vous préciser la raison pour laquelle vous avez employé le terme "paracinéma" et aborder plus spécifiquement le lien que celui-ci entretient avec le «cinéma élargi »? Est-il possible de déterminer quel terme prime, de la performance ou du concept, de la pratique filmique ou de la «théorie du dispositif»?

\section{Ken Jacobs}

Gene Youngblood a écarté mon travail dans son ouvrage qui porte sur le "cinéma élargi» [Expanded Cinema, Dutton, 1970] ; mais je n'avais pas cela à l'es-

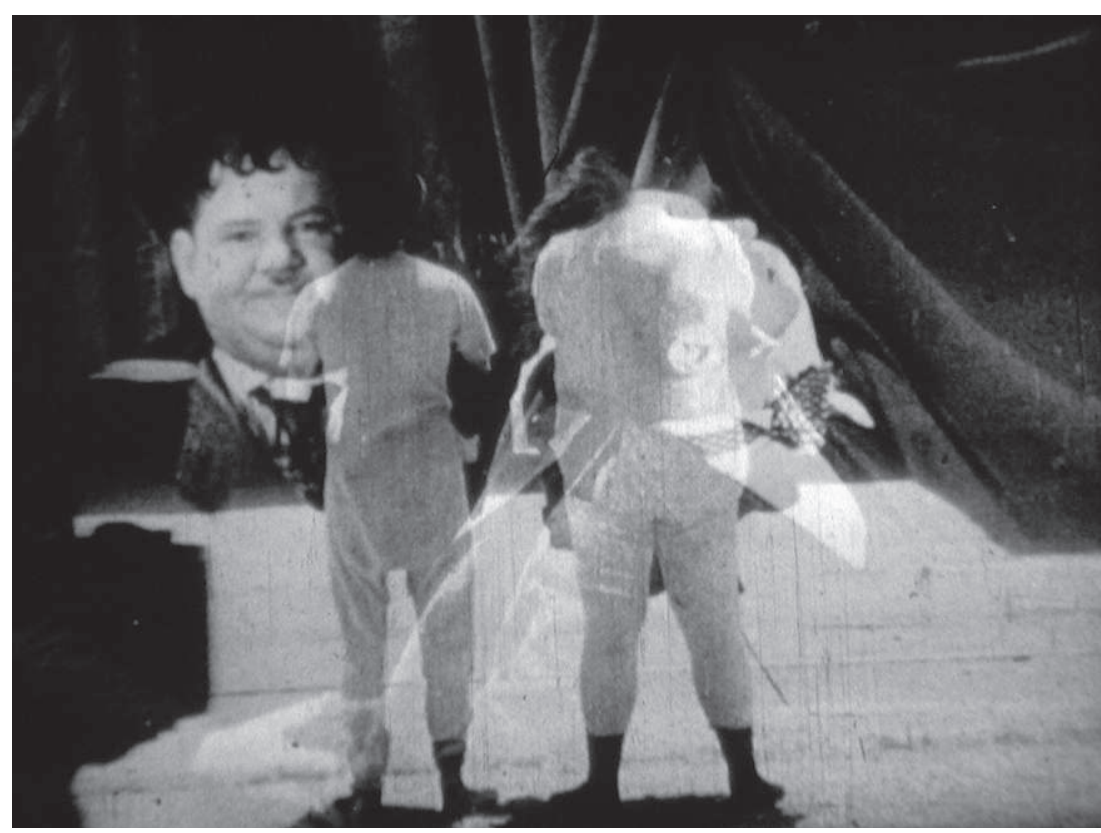

Ontic Antics Starring Laurel and Hardy (1997/2005), Ken Jacobs (Nervous System Performance) 
prit, je voulais alors indiquer une différence essentielle entre le cinéma traditionnel et ma propre démarche. Conventionnellement, l'acte de création cinématographique prend fin avant le stade de la projection: le travail est «dans la boîte». Mais dans The Nervous System, et par la suite dans The Nervous Magic Lantern, le projecteur - ou plus d'un projecteur - devient le lieu de la création filmique. Ce qui avait été filmé est élargi durant la performance de façon imprévisible, de telle sorte que ni notre vécu, ni le travail préalable sur le tournage, ni même mon travail derrière les machines ne permettent d'anticiper le spectacle - et ceci, jusqu'à ce que je voie sur l'écran les effets en mouvement suscitant une illusion de relief. Cela dépend de la mise en place particulière du dispositif au moment de la projection : c'est-à-dire des faisceaux des deux projecteurs, de la façon dont ils se superposent à l'écran, et de la position et relation respective des projecteurs. L'image à l'écran suggère des changements physiques qui permettent de transformer les choses et de me surprendre; qu'importe le nombre de répétitions, au moment de la projection je tâtonne dans l'inconnu, j'avance instinctivement.

L'expérience visuelle des spectateurs ne peut pas être anticipée; si nous allions au cinéma pour réfléchir à la vie, cette expérience constituerait une désespérante sortie hors de notre univers familier. Mais l'aventure permanente à laquelle nous invitons les gens, Flo [Florence Jacobs, son épouse] et moi, est immédiate, s'emparant du spectateur sur place, au moment même où il assiste à ce qui se déroule sur l'écran.

J'ai parlé de paracinéma afin de désigner une démarche qui rompt avec les attentes convenues: parallèlement aux procédures standard, une façon différente de procéder. Ce qui en retour a impliqué d'autres gains que ceux escomptés, entièrement sensoriels en ce qui me concerne (comprenant un jeu de dérèglement de l'équilibre physique!). En tant qu'artiste actif, je suis anti-conceptuel; ce qui est bien et même nécessaire pour l'étude de l'art mais qui ne peut d'aucune manière s'y substituer, tout comme les manuels de sexe constituent un mauvais substitut de

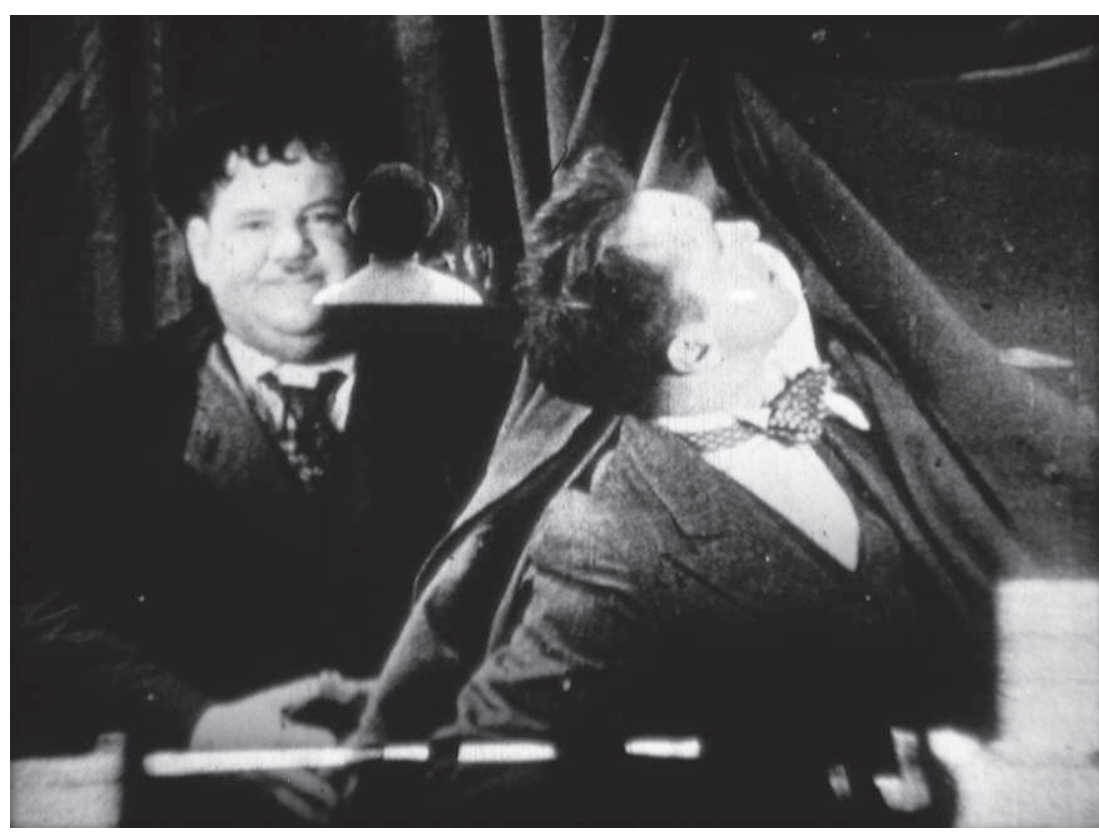

Ontic Antics Starring Laurel and Hardy (1997/2005), Ken Jacobs (Nervous System Performance) 
l'acte sexuel. Ces derniers temps, les visites dans les musées et les galeries s'apparentent trop à des manuels de sexe, à mon goût.

Pour apprécier l'expérience que je propose, il faut être prêt à se jeter dans l'inconnu et à faire face à l'impossible. Nos sens sont sur un mode automatique. Ils peuvent cependant être merveilleusement induits en erreur. Dès lors que nous reconnaissons le fait que nos sens peuvent être induits en erreur, il y a d'autres planètes qui s'offrent à nous, à portée de main et immédiatement, avec pour billet d'entrée le cinéma d'artistes.

De la modestie avant tout, à propos de ce que l'on croit savoir!

\section{Décadrages}

Vous avez mentionné un mode de vision qui joue sur un dérèglement physique, qui peut impliquer une désorientation sensorielle. Pourriez-vous développer cet aspect? Quels sont les effets prémédités et quels sont ceux qui résultent directement de vos perfor- mances? Pourriez-vous illustrer plus précisément ces procédés par rapport à vos performances pour le Nervous System et le Nervous Magic Lantern?

\section{Ken Jacobs}

Je reviendrai par la suite sur ce dernier point, mais pour commencer je dirais que Jésus ou l'entreprise de Jésus n'a pas imposé la croix aux gens du jour au lendemain, venant de nulle part; c'est une préoccupation inhérente à l'esprit humain que de rechercher et de déterminer l'axe vertical-horizontal. Le fait est que le crucifix historique ressemblait davantage à une svastika qu'à une croix, non? Le mouvement, l'excitation engendrent toujours un équilibre instable. Cézanne, le muet, a fait d'excellents tableaux en inclinant les verticales afin qu'elles se distinguent des limites latérales ou des cadres de ses tableaux. Le cinéma incarne cette tension. Je voulais m'extraire du règne de la verticalité carrée et réaliser des œuvres qui se déploient dans le temps - comme c'est le cas avec la musique et qui ne se stabilisent qu'à la fin. Il est difficile, en

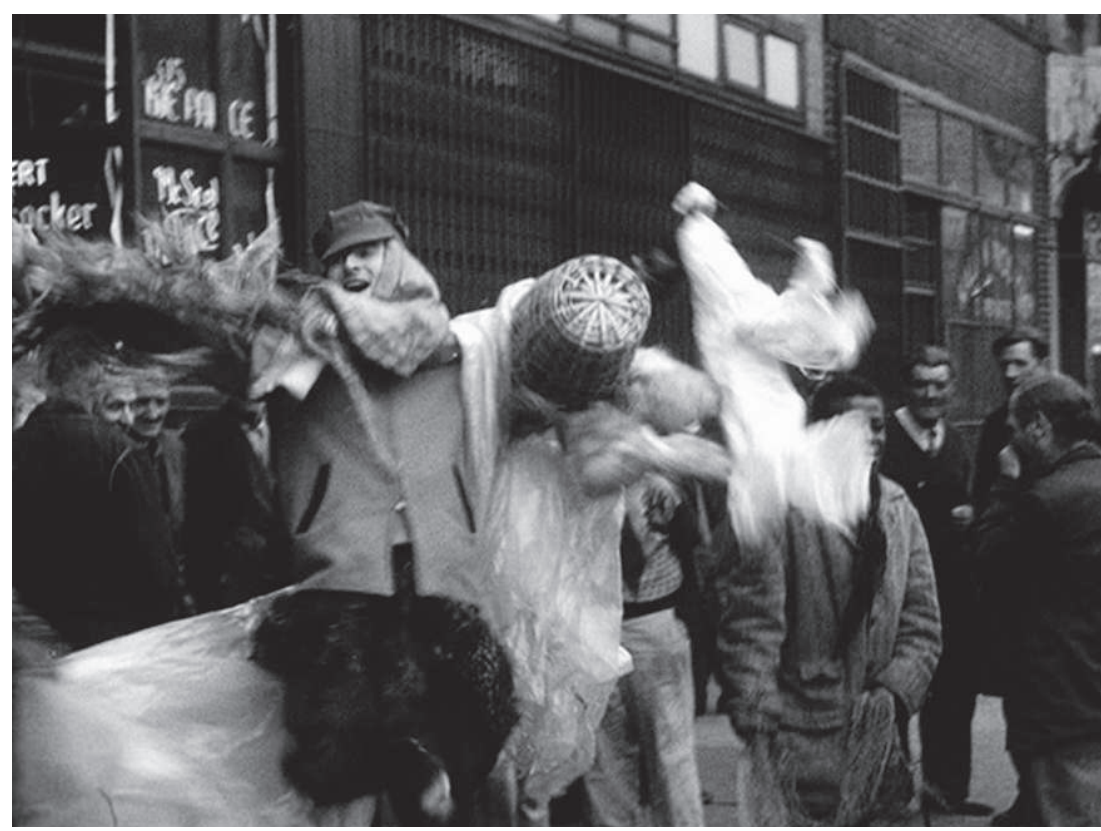

Two Wrenching Departures (1989/2006), Ken Jacobs (Nervous System Performance) 
regardant à travers l'objectif d'une caméra, de ne pas immédiatement composer l'image et de ne pas se propulser par la suite d'un point pictural statique au suivant. Les histoires au cinéma créent un suspense et maintiennent le public dans un état de déséquilibre par le biais de questions qui sont progressivement résolues. Ce n'est pas suffisant, le cinéma n’y parvient pas, seuls les films des premiers temps y parvenaient.

\section{Décadrages}

Pour en revenir à vos performances avec le Nervous System et le Nervous Magic Lantern, pourriezvous nous en dire davantage sur votre travail avec la chronophotographie, les films primitifs et le cinéma des premiers temps? Contre l'entreprise de Jésus, quelle est la fonction du flicker qui suscite une instabilité au sein de l'œuvre et chez le spectateur?

\section{Ken Jacobs}

Le flicker... c'est quelque chose, selon mon expérience, qui a été découvert par Alfons Schilling de Vienne [artiste et cinéaste né à Bâle en 1934, lié à l'actionnisme viennois dans les années 1950 et qui s'établit à New York de 1962 à 1986], qui habitait dans le coin à Manahattan durant les années 1970 et qui est la seule personne que je connaisse à réaliser un travail intéressant en $3 \mathrm{D}$. Nous échangions nos découvertes techniques et parfois du matériel. J'avais produit des effets de profondeur dans l'imagerie 2D à ses débuts grâce à un mécanisme d'obturation que j'avais inventé qui alternait (à travers un changement extrêmement lent) des vues de photogrammes adjacents au sein de deux copies de film identiques. Ma fascination pour le cinéma des origines remonte à Tom, Tom, The Piper's Son [film de 1905 de Billy Bitzer, restauré avec l'aide de Ken Jacobs à partir d'un paper-print, et point de départ du film éponyme de Jacobs en 1969], à la fin des années 1960. Ce qui m’a amené à m'intéresser à la période précédente, où l'enregistrement des mouvements de la vie constituait le centre d'intérêt, bien avant que le mode narratif ne l'emporte. Je me considérais moi-même comme

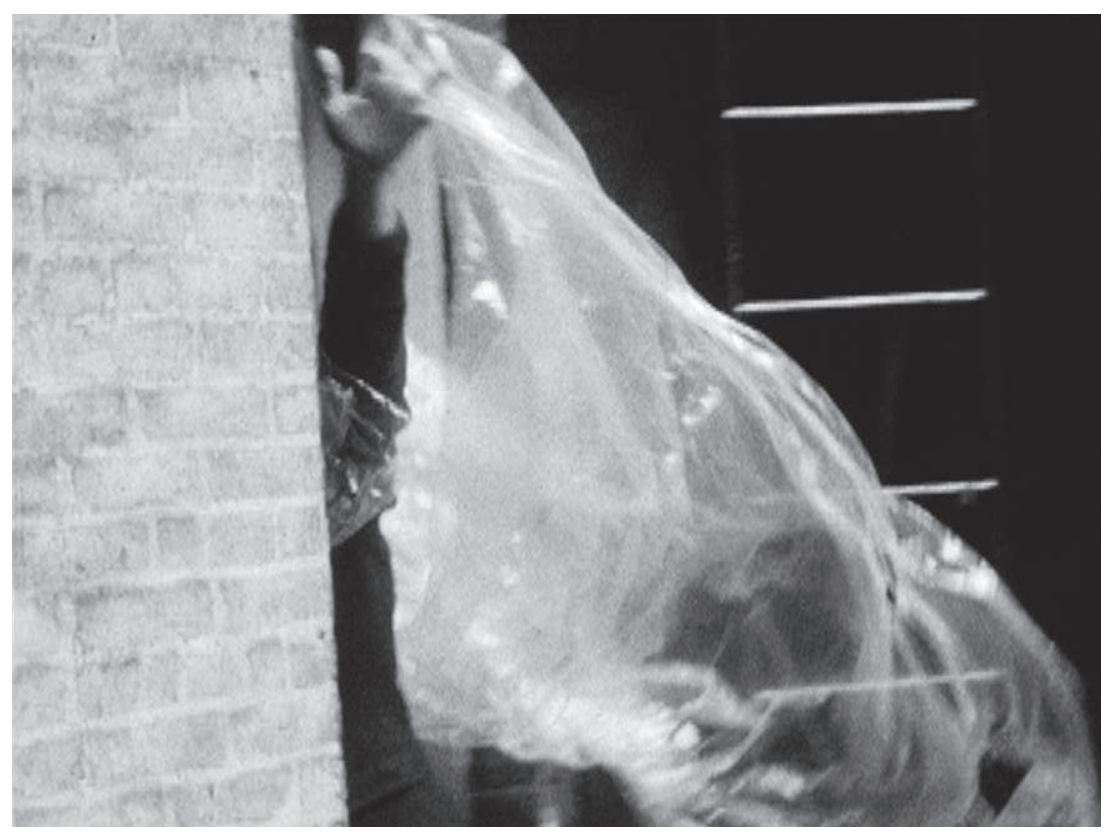

Two Wrenching Departures (1989/2006), Ken Jacobs (Nervous System Performance) 
l'un de ces types, l'un de ces inventeurs, à cette différence près que mon intérêt s'était déplacé sur le travail du cerveau lui-même et sur sa capacité à être merveilleusement induit en erreur. Alfons, qui travaillait avec des diapositives couleur stéréoscopiques, tentait d'obtenir mes effets de profondeur grâce à un système plus simple d'alternance (sans lunettes), à savoir l'obturateur rotatif, fixé devant un projecteur de diapositives 3D. Le flicker s'était immiscé dans le dispositif! Et des évènements d'une densité inimaginable commençaient à se produire. Il m'avait encouragé à employer ce modèle très ancien d'obturateur, et mon travail filmique a progressé. Mon propre système d'obturation avait créé des formes plutôt plates dans un espace en profondeur; mais désormais, on voyait des formes rondes et voluptueuses (qui pouvaient être animées et remodelées). J'ai réalisé une œuvre intitulée SCHILLING pour rendre hommage à ce don, et j'ai ensuite travaillé sur $X C X H X E X R X R X I X E X S X$ à partir d'un film pornographique français muet.
Le flicker «fusionne» les photogrammes. Le tic-tac du temps a disparu; une scène unique tridimensionnelle et en mouvement est apparue, qui peut être maintenue aussi longtemps qu'on le désire; ce qui signifie que le projectionniste la dirige là où il veut et aussi longtemps qu'il le souhaite, en un cheminement continu sans pour autant que celui-ci doive aboutir où que ce soit. Essayez cela dans la vraie vie. La façon dont les deux images se chevauchent sur l'écran, leurs différences de taille, peut-être par rapport à la verticalité, suscitent l'émerveillement. Ce qui constitue un développement alternatif à la représentation de la vie qui avait précédé l'essor du cinéma.

Alphons fut passablement agacé par l'accueil réservé à mon travail avec l'obturateur rotatif. Ce n'était pourtant pas grand-chose, une expérience isolée en décalage avec la totalité du champ de la 3D («la 3D stupide») qui se dirigeait dans d'autres directions, mais il m'a interdit de l'employer à nouveau, ou du moins tenta de le faire. Car il était trop tard. J'avais breveté l'intervalle noir dans son utilisation digitale,

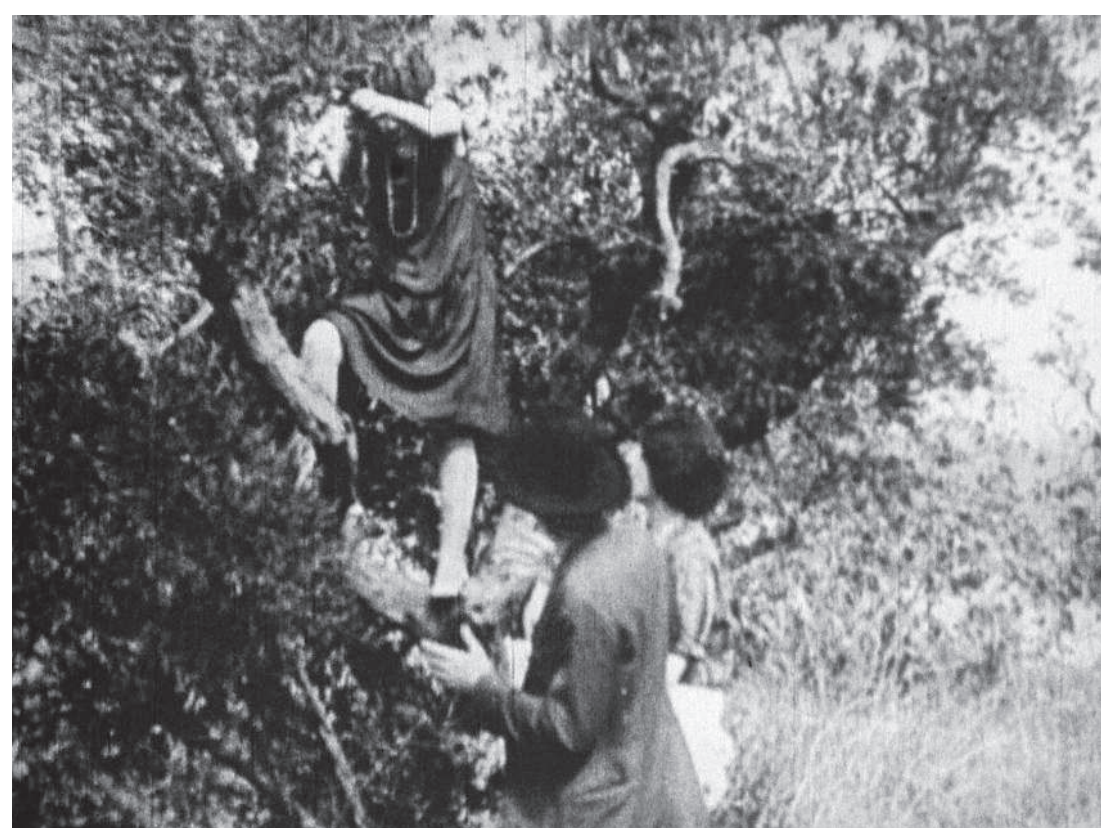

XCXHXEXRXRXIXEXSX (1980), Ken Jacobs (Nervous System Performance) 
et j’ai réalisé nombre d'œuvres numériques depuis en employant cette technique.

\section{Décadrages}

Comment reliez-vous votre intérêt pour les erreurs de perception du cerveau et les effets induits sur le corps? D'une part, nous pourrions presque parler d'imagerie mentale, ou en tout cas d'images subjectives; d'autre part, le choc du flicker provoque des vertiges, un état d'épilepsie presque. Pourriez-vous commenter cette relation, voire cette opposition?

\section{Ken Jacobs}

Nous, Flo et moi, et certaines de nos connaissances, sommes devenus de bons amis du flicker. Phil Solomon a pu parler de la difficulté à s'adapter au cinéma dépourvu de flicker, qui paraît en effet terriblement boiteux. Si l'on ne parvient pas à suivre l'histoire, rien n'est plus ennuyeux que la surface inerte du cinéma standard. Je réalise les films qui m'intéressent, et je n'ai aucune expérience de vertiges ni d'épilepsie. Il existe pourtant des cerveaux qui ne supportent pas le flicker; et j'ai perdu un étudiant doué pour avoir oublié de l'avertir du danger. L'artiste doit néanmoins travailler à partir de sa propre expérience, et la musique existe même s'il y a des sourds.

Le suspense à la Hitchcock n'existe qu'à l'intérieur du monde de la fiction. La question: "Que vat-il se passer ensuite?» n'est pas ce qui m'intéresse le plus au cinéma - alors que le présent nous échappe ou résiste à une saisie rapide. Apparemment, nous ne sommes pas encore rassasiés du carton The End. Je veux du suspense viscéralement. C'est l'état de tension qui se joue lorsque nous nous approchons ou que nous nous éloignons du point d'équilibre. Un équilibre stable est un équilibre mort; ce dont nous avons besoin et qui force notre admiration, c'est un équilibre mis en péril. Il est difficile d'admirer quelqu'un qui est enraciné dans le sol, bien que dans mon travail avec l'image stéréoscopique je deviens de plus en plus empathique avec les arbres et leur relation différente au temps.

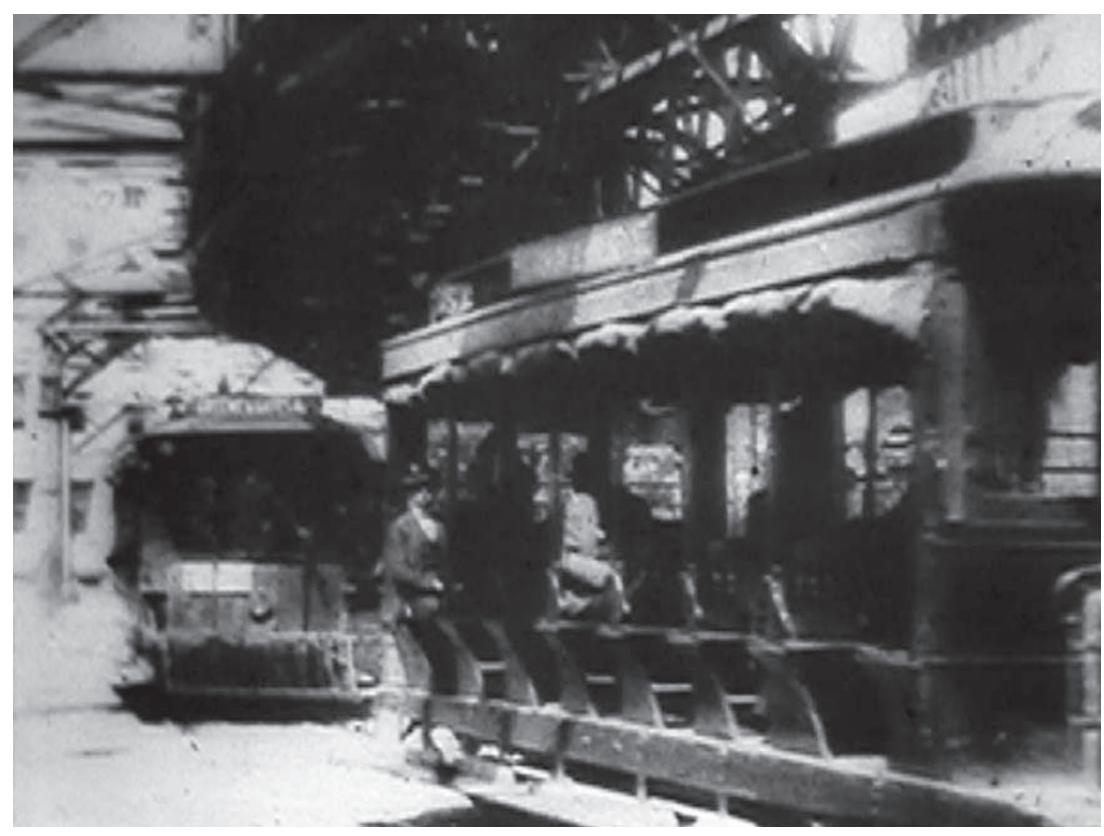

New York Street Trolleys 1900 (1997/1999), Ken Jacobs (Nervous System Performance) 
Mon cinéma pâtit d'un équilibre stable, peutêtre parce que je viens de la peinture et non pas de la musique, où l'on peut par jeu s'attacher à la tonique. Je ne peux pas m'empêcher de composer un cadre équilibré lorsque je regarde à travers l'objectif d'une caméra; alors, mon travail récent tente de se déprendre de cet ordre-là. Je peux d'une certaine manière imaginer un travail cinématographique réalisant son équilibre au fil du temps, où la première image serait en fin de compte renforcée par la dernière, mais je peux aussi dire: "Au diable, l'équilibre!» Ce serait d'ailleurs beaucoup d'efforts pour pas grand-chose. Si le sujet de l'œuvre n'est pas réduit à une alternance de lumière/non-lumière, bruit/non-bruit, comme chez Kubelka [Arnulf Rainer, 1958-1960], le seul semblant d'ordre qui vient à s'imposer, c'est la normalité, ce qui nous semble être familier, c'est-à-dire moins de LSD.

Il en va de même avec les erreurs de perception, qui peuvent être amusantes et nous enseigner la modestie face à la connaissance de La Vérité Des Choses. La religion c'est du pipeau, et la philosophie tourne court. La simple chance d'être nés dotés d'une bonne constitution qui résiste à un régime constant de stupéfaction permet de nous en sortir.

(Traduit de l'américain par Sylvain Portmann) 